\title{
Consequences of lower extremity and trunk muscle fatigue on balance and functional tasks in older people: A systematic literature review
}

\author{
Jorunn L Helbostad ${ }^{1,2^{*}}$, Daina L Sturnieks ${ }^{3}$, Jasmine Menant ${ }^{3}$, Kim Delbaere ${ }^{3,4,5}$, Stephen R Lord ${ }^{3}$, \\ Mirjam Pijnappels ${ }^{6}$
}

\begin{abstract}
Background: Muscle fatigue reduces muscle strength and balance control in young people. It is not clear whether fatigue resistance seen in older persons leads to different effects. In order to understand whether muscle fatigue may increase fall risk in older persons, a systematic literature review aimed to summarize knowledge on the effects of lower extremity and trunk muscle fatigue on balance and functional tasks in older people was performed.

Methods: Studies were identified with searches of the PUBMED and SCOPUS data bases.

Papers describing effects of lower extremity or trunk muscle fatigue protocols on balance or functional tasks in older people were included. Studies were compared with regards to study population characteristics, fatigue protocol, and balance and functional task outcomes.

Results: Seven out of 266 studies met the inclusion criteria. Primary findings were: fatigue via resistance exercises to lower limb and trunk muscles induces postural instability during quiet standing; induced hip, knee and ankle muscle fatigue impairs functional reach, reduces the speed and power of sit-to-stand repetitions, and produces less stable and more variable walking patterns; effects of age on degree of fatigue and rate of recovery from fatigue are inconsistent across studies, with these disparities likely due to differences in the fatigue protocols, study populations and outcome measures.

Conclusion: Taken together, the findings suggest that balance and functional task performance are impaired with fatigue. Future studies should assess whether fatigue is related to increased risk of falling and whether exercise interventions may decrease fatigue effects.
\end{abstract}

\section{Background}

Loss of functional ability and impaired balance control in older people is well documented and attributed to age-related sarcopenia and sensorimotor decline. Another factor, likely to further affect an older person's functional abilities is muscle fatigue. Studies in young people show that muscle fatigue in the lower limbs increases postural sway $[1,2]$, that fatiguing postural back muscles impairs head and trunk control while walking [3], and that quadriceps muscle fatigue alters gait parameters related to slip propensity [4].

* Correspondence: jorunn.helbostad@ntnu.no

'Department of Neuroscience, NTNU, Trondheim, Norway

Full list of author information is available at the end of the article
Effects of fatigue of specific muscle groups on muscular performance (e.g. strength) in both young and older people has been investigated extensively (see Kent-Braun 2009 [5] for review). Interestingly, despite typical loss in muscle strength with increasing age, several studies have demonstrated that older people are more resistant to muscle fatigue than young people following isometric and dynamic lower extremity muscle work [6-14]. Possible mechanisms for this include lower maximal motor units discharge rates, slower contractile properties and greater reliance on oxidative metabolism in older people [5]. However, not all research supports relative muscle fatigue resistance in older persons $[15,16]$. In addition, fatigue resistance in older people is not evident for the trunk muscles [17], 
and one study showed recovery rate after fatigue to be slower in older fallers compared to older non-fallers and young adults [13].

In order to understand whether muscle fatigue in older persons affects fall risk, greater focus should be on consequences for balance and functional performance. Therefore, this systematic literature review assessed the effects of lower extremity and trunk muscle fatigue (seen as a decrement in performance following exercise) and recovery on balance and functional tasks in older people and synthesized the findings with regard to how age, gender, fatigue protocols and effect of fatiguing different muscle groups affect balance and functional abilities. This knowledge may assist in designing falls risk assessments and exercise programs in clinical settings for older people.

\section{Methods}

\section{Search strategy and selection criteria}

We conducted a systematic literature review until June 2009, with a primary search in the PUBMED and a secondary search in the SCOPUS databases. The PUBMED search was performed for the MeSH term "Muscle Fatigue" and the search limited to Human studies, English language, and subsets of Aged 65+ and 80+. The SCOPUS search used the following terms: TITLE(fatigue AND (muscle OR physical)) AND KEY(aging OR ageing OR older OR elder* OR aged) AND (LIMIT-TO(DOCTYPE,"ar")) AND (LIMIT-TO(LANGUAGE,"English”)). Additionally, we searched for the terms "fatigue" AND "older" in relevant journals within gerontology and geriatric medicine (Journals of Gerontology and Journal of the American Geriatric Society) and physiology (European Journal of Applied Physiology, Journal of Applied Physiology, Muscle and Nerve and Electromyography and Clinical Neurophysiology). Duplicates between searches were removed.

\section{Inclusion criteria and quality assessment}

To be included, papers had to describe muscle fatigue protocols for the lower extremities or trunk muscles (except diaphragm or pelvic floor muscles). Papers were excluded if they were reviews, methodological or descriptive papers, the focus was on patient groups, all participants were younger than 50 years of age, fatigue was only measured by self-report, or studies were performed in animals or in vitro. Finally, papers reporting effect of muscle fatigue on local muscles and not on balance or functional tasks were also excluded.

\section{Abstraction of data and analysis}

Five authors contributed to the review process. Every title and abstract was screened by two randomly allocated reviewers, using the inclusion criteria. A full paper review was performed for the remaining publications by two other randomly allocated reviewers. For the included papers, a first reviewer reported on study aims, population, fatigue protocol, how fatigue was measured, and specification of outcomes and on findings. A second reviewer provided additional details as deemed necessary.

For descriptions of outcomes we divided balance and functional tasks into quiet standing, voluntary movements or external perturbations.

\section{Results and Discussion \\ Search Results \\ Studies identified}

The PubMed-search revealed 130 hits and the Scopussearch 170 hits, of which 37 were duplicates. Additionally, three papers were detected through searching selected journals, leaving 266 papers for abstract review. Following abstract review 210 papers were excluded, leaving 56 papers for a full review. Of these, 36 papers reported fatigue of lower extremity or trunk muscles in older people. However, 29 did not have a balance or a functional task outcome, leaving seven papers for final inclusion [18-24].

\section{Study populations}

Study populations, fatigue protocols and outcome measures are summarized in Table 1. Five studies included a sample of older people in addition to a control group; four young [18-21] and one older [22] control group. Two studies had no control group [23,24]. The sample sizes ranged from 11-24 participants. Mean age of the older people varied from 62.2 to 78.2 years, with a range across studies from 55 to 86 years. One study included only women [23], one only men [20], and five both genders $[18,19,21,22,24]$, of which only Petrella et al. [21] examined gender differences.

All studies involved healthy community-dwelling older participants without any musculoskeletal disorders, except for Helbostad et al. [22], who reported prevalence of eleven diseases with $45 \%$ of participants having hypertension, $41 \%$ incontinence, and $36 \%$ stroke or osteoporosis. Helbostad et al. further reported of $41 \%$ with a fall the previous year, while two other studies explicitly stated that participants had no history of falls in the past year $[18,19]$. Participants in the study by Mademli et al. [20] were active in sports, while two studies explicitly reported exclusion of participants engaged in resistance training during the past six months [24] or five years [21].

\section{Fatigue protocols and outcome measures}

In five studies, the fatigue protocol was performed on one or more specific muscle groups using a dynamometer, at a load related to the participants' maximum voluntary capacity (MVC) [18-20,23,24]. In two studies, 


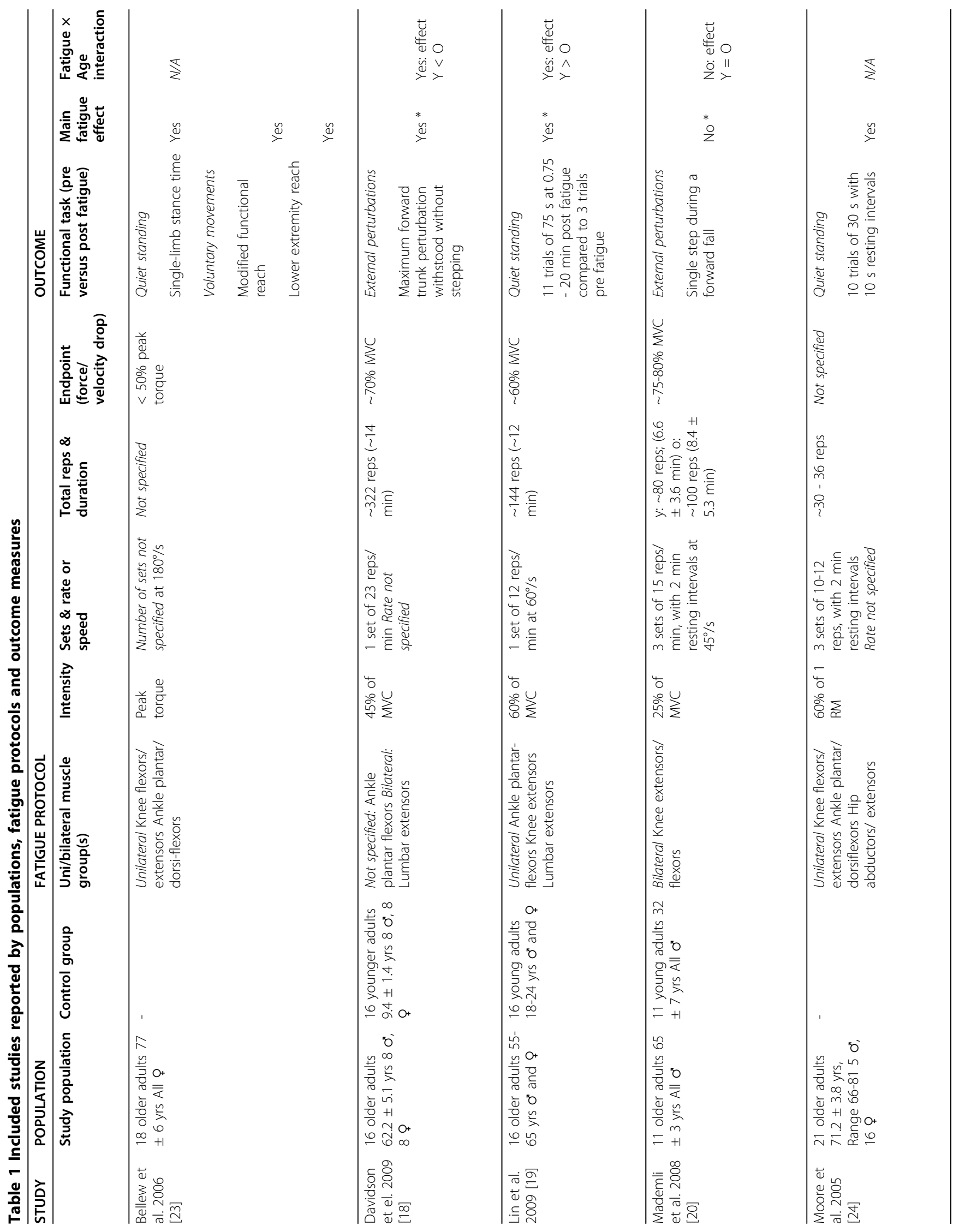


lower limb muscle fatigue was performed by sit-tostand repetitions [21,22]. Participants were instructed to cross arms and repeatedly rise from a chair to an erect position at a fast speed, then sit down either at a fast [22] or slow speed [21]. While Petrella et al. [21] examined fatigue effects over 10 sit-to-stand repetitions, Helbostad et al. [22] required participants to perform sit-to-stand trials until they felt too exhausted to continue.

Four studies fatigued muscle groups over multiple joints $[18,19,23,24]$ with three examining differential effects between the muscle groups $[18,19,23]$. Five studies sought to fatigue knee-flexors and/or extensors [19-21,23,24], three ankle plantar- and/or dorsi-flexors $[18,19,23,24]$, one hip-abductors and extensors [24], and two fatigued lumbar extensors $[18,19]$. The two studies employing sit-to-stand repetitions [21,22] did not specify muscle groups that were fatigued.

Three studies fatigued one or more specific muscle group unilaterally in the dominant leg [19,23,24], despite the outcome measure being two-legged standing. Four studies used a bilateral fatigue task to study effects on voluntary movements including sit-to-stand [21], walking [22] and balance recovery from external perturbations $[18,20]$.

For the five studies using isokinetic contraction fatigue protocols [18-20,23,24], force levels varied from 25-60\% MVC. Contraction velocity was specified in three studies and ranged from $45-180^{\circ} / \mathrm{s}[19,20,23]$. Number of repetitions for the sit-to-stand protocols were 10 [21] and $\sim 25$ [22], while other exercises employed between $\sim 30$ [24] and 322 [18] repetitions. Time to perform the fatigue protocol was reported for three studies [18-20] and varied between 8.4 and 14 minutes.

The endpoints of fatigue protocols reported were either: participants unable to lift a weight through the whole range of motion [20,24], performance dipped below a pre-defined threshold over three consecutive repetitions $[19,23]$, participants were apparently exhausted [22], or were based on a pre-defined number of repetitions [21] or time duration [18]. The relative force decline at the endpoint, explicitly reported in two studies $[18,23]$ and assumed in two others $[19,20]$, varied between 50 and $70 \%$ of maximum pre-fatigue force level. One study [21] described the effect of fatigue in terms of velocity reduction over repetitions, while another did not specify the fatigue endpoint [24].

Outcome measures varied between studies. Three studies examined quiet stance, during which Lin et al. [19] and Moore et al. [24] calculated centre of pressure (COP) profiles, while Bellew et al. [23] measured single limb stance time. Three studies involved voluntary movements as outcome measures: upper and lower 
extremity reach length [23], sit-to-stand performance [21] and walking at preferred gait speed [22]. Two studies examined balance recovery from external perturbations in standing: sudden release from a forward leaning position [20] and a perturbation applied to the upper trunk [18].

Only one study examined effects of recovery rate on balance performance [19].

\section{Results on Fatigue Effects}

This systematic literature review examined the effects of lower limb and trunk muscle fatigue on balance and functional tasks in older people. Since none of the manuscripts aimed to study the specific mechanisms of fatigue, fatigue was defined as a decrement in performance following exercise. Six out of seven studies found a significant effect of fatigue on balance in older people [18,19,21-24] while one showed no effect [20]. The studies varied considerably in study populations, fatigue protocols and outcome measures.

\section{Main effects of fatigue}

Three studies demonstrated that balance performance during quiet stance was poorer after fatigue, as evidenced by reduced single limb stance time [23] and increased postural sway $[19,24]$. Single limb stance time was reduced following repeated isokinetic knee-flexion/ extension and dorsi/plantar-flexion exercises [23]. Similarly, postural sway was increased, evidenced by increased COP displacement while standing following repeated hip-, knee- and ankle-flexion/extension resistance exercises [24]. Finally, a detrimental effect of fatigue on various postural sway parameters was found after fatiguing the ankle plantar-flexors, knee-extensors, lumbar-extensors and shoulder-extensors [19]. The amount, speed and frequency parameters of postural sway are commonly used indicators of postural stability, while single leg stance time is a simple measure of postural stability while balancing on one leg. Taken together, these results suggest that fatigue via resistance exercises to lower limb and trunk muscles generally induces postural instability during quiet standing.

Three studies demonstrated that balance performance during voluntary movements was poorer after fatigue, as evidenced by impaired functional reach [23], reduced speed and power of sit-to-stand repetitions [21] and altered gait parameters [22]. This fatigue-induced increase in step width, medio-lateral trunk accelerations and variability in antero-posterior acceleration is related to decreased walking stability and is comparable to gait patterns in frail people [22]. These studies suggest that induced fatigue of hip, knee and ankle muscles impairs functional reach [23], reduces the speed and power of sit-to-stand repetitions [21] and produces less stable and more variable walking patterns [22].
Contrasting results were found for the effects of fatigue on balance reactions to unexpected perturbations $[18,20]$. Davidson et al. [18] found balance recovery, following a perturbation to the trunk, was impaired after fatiguing ankle plantar-flexors and lumbar-extensors. Conversely, Mademli et al. [20] showed no effect of knee flexor and extensor fatigue on balance recovery following a sudden release from a supported leaning posture after fatigue exercise at $25 \%$ MVC. However, the lack of effect in the latter study might be explained by the fatigue protocol which involved a low relative force or sub-optimal protocol relative to outcome measure (i. e. the knee muscles fatigued but the hip muscles are required for balance recovery) may explain the lack of a fatigue effect in the latter study.

\section{Effect of age}

Four studies [18-21] examined whether fatigue effects on balance and functional tasks differed between young and older people. No age-related fatigue differences were reported for responses to external perturbations [20] and sit-to-stand performance [21]. Davidson et al. [18] examined 13 different balance response variables (perturbation magnitude withstood and a range of COM and COP measures) following an unexpected perturbation. No age-related differences were found for 10 variables. However, compared to young, older people had significantly increased COP peak velocity, time-to-peak velocity and time-to-return to the original COP position following the perturbation in the fatigued state. These findings suggest that, relative to young, older people's balance was more challenged by the perturbation following fatigue. In contrast to this, Lin et al. [19] found postural sway during quiet standing to be more affected by fatigue in young, compared to older people. These contrasting results could indicate that different strategies may be adopted in young and older people in static and dynamic balance tasks with fatigue (for example, older people might rely more on a hip strategy to control postural sway under fatigued conditions compared to young). Further work is required to test this hypothesis.

Several studies investigating muscle fatigue on muscle strength and function have suggested that older people are more fatigue-resistant than younger people $[8-14,25]$. Three mechanisms have been proposed. First, the majority of these studies suggest that fatigue resistance in older people is likely due to morphological mechanisms, rather than central or peripheral activation changes. Older people have relatively less Type II (fasttwitch/fast-fatiguing) muscle fibres compared to young people [5]. Second, Mademli and Arampatzis [11] have suggested that older people have shorter fascicle length (they found a strong relationship between time to fatigue and muscle fascicle length) and lower ratio of active muscle volume and force production, which could be an 
energetic advantage. Third, metabolic mechanisms were proposed by Lanza et al. [7] who studied glycolytic ATP production in young and older people and concluded that older people who fatigued relatively slower, had improved metabolic economy (less metabolite accumulation).

Some studies have not shown significant age-related differences in fatigue [26-28], which might be explained by differences in fatigue protocols, outcomes or sample characteristics. McNeil et al. [27] found older people to be more fatigable than young during a velocity-dependent power task and suggest their contrasting findings are due to the more functionally relevant task, which employed a lighter load, and is more comparable to daily life tasks.

Explanation for the differing findings may relate to the complexity of the various processes that contribute to muscle fatigue in older people, such as decline in mitochondrial function, alterations in brain neurotransmitters, oxidative stress and inflammation [29]. The underlying mechanisms for muscle fatigue, particularly in old age are still poorly understood, and future studies are required in this area. Further research is also required to elucidate the roles played by health and fitness, the type of muscle work performed and the particular muscle groups fatigued in influencing balance control and functional task performance.

\section{Effects of fatiguing different muscle groups}

Knee-extensors were fatigued in six studies [19-24], of which four consistently showed consequential detrimental effects on balance and functional tasks [19-24]. This suggests that knee-extensors are important in controlling balance during quiet stance and functional tasks [21-23]. Two studies did not show a knee extensor fatigue-effect, which could be explained by the employed fatigue protocol $[20,21]$. The protocol of Lin et al. fatigued only one leg, which could explain why there was no effect on bilateral quiet standing [19]. The fatigue protocol of Mademli et al. involved long rest intervals (two minutes) between sets, which was not sufficiently fatiguing to find a significant reduction in muscle force [20]. Additionally, the lack of effect of knee flexor/extensor fatigue on balance recovery from a sudden release of a supported leaning posture might be explained by the fact that recovery from a leaning position predominantly involves hip flexor and/or ankle plantar-flexor muscles, more so than knee muscles [20].

Ankle plantar flexors were fatigued in four studies, of which all indicated that fatigue has a detrimental effect on standing balance, as evidenced by an increased postural sway during quiet standing $[19,23,24]$, reduced reach [23] and reduced balance recovery following external perturbation while standing [18]. The effect of plantar-flexor fatigue on balance performance is apparent, since standing balance is regulated primarily by the ankle plantar-flexors. Furthermore, forward reaching [23] and resistance to forward postural perturbations [18] involve a large degree of plantar-flexor activity due to their antigravity action to prevent forward falling.

Lumbar extensor muscles were fatigued in two studies which both indicated that fatigue has a detrimental effect on standing balance, evidenced by increased postural sway during quiet standing [19] and increased peak COP velocity following external perturbations while standing [18]. Lumbar extensors would provide resistance to the anterior perturbations used in this study, by arresting the forward momentum of the upper body induced by the perturbation. However, the large number of outcome parameters also makes it possible that the significant effect on peak COP velocity was by chance. The study by Lin et al. [19] consistently (across multiple balance parameters) found more acute effects on standing balance from fatigue of the lumbar extensors, compared to knee-flexors and plantarflexors. The authors suggested that back muscle fatigue might impair lumbar position sense, as well as ankle joint motion sense. This is in line with findings from Pline et al. [30] who reported that lumbar extensor fatigue may impair central processing of ankle proprioceptive signals, leading to poorer balance control. In addition, balance changes may have been more sensitive to back muscle fatigue, as this was induced bilaterally, compared to the knee and ankle muscles which were fatigued unilaterally.

Only one study investigated effect of fatigue of hip muscles on balance [24]. However, because hip abductors and extensors were fatigued in combination with muscles from other lower limb joints, it is not possible to assess the contribution that hip muscle fatigue alone had on postural sway. Further research is required to provide more insight into the role of hip muscle fatigue on balance and functional task performance.

\section{Gender differences}

Only one study analysed men and women separately [21]. Even though sit-to-stand power over repetitions declined more in men (29\%) than women (16\%) [21], however, no interaction of gender and age was reported. Hicks and McCartney [31] have suggested that women might be more fatigue resistant than men due to energy costs associated with the ratio of length-to-cross-sectional area of the muscle (women have shorter muscle length relative to crosssectional area) or differences in the distribution of fibre type (women may have fewer Type IIB fibres). Further research is required to draw more general conclusions on gender in the effects of fatigue on balance and functional tasks.

\section{Recovery from fatigue}

Only one study investigated fatigue recovery rates, and indicated recovery from muscle fatigue was slower in older people compared to young [19]. However, 
conclusions from this study should be drawn with caution, as significant effects of muscle fatigue on quiet standing were only seen in young and not in older people. Häkkinen [8] studied recovery over 60 minutes from bilateral leg press exercise in 23 young, middle-aged and older women and found slower recovery in older people, despite a lower level of induced fatigue, relative to the younger groups. Slower recovery may put older people at risk of balance loss for a longer period. More research on the effects of recovery from fatigue for balance and functional tasks in older adults and how it relates to fall risk is required.

\section{Conclusions}

Despite variations in study design, populations, fatigue protocols, and outcome measures findings were fairly consistent in that fatigue of the lower extremity and trunk muscles impairs balance and performance of functional tasks. This suggests that fatigue protocols could be useful in the prediction of falls; however more studies are required to decide whether muscle fatigue should be included in routine clinical fall risk assessments. More specifically, future studies should employ a common exercise to systematically examine fatigue effects of each muscle group on typical balance tasks (such as quiet standing, walking, sit to stand and responding to unexpected perturbations). This would elucidate relative effects of the different muscle groups on balance performance and assist comparisons across studies. In addition, the benefits of exercise on muscle strength and power and on recovery rate after fatigue need further investigation to indicate whether specific exercises to improve muscle fatigability should be incorporated in exercise programmes to prevent falls.

\section{Acknowledgements}

Jorunn L Helbostad was financially supported by a grant from the Health Authorities in Mid Norway, Daina Sturnieks by an NHMRC Population Health Capacity Building Grant in Injury Prevention, Trauma and Rehabilitation, Jasmine Menant by an NHMRC Population Health Capacity Building Grant (\# 568940), Stephen Lord by an NHMRC Senior Principal Research, and Mirjam Pijnappels by the Netherlands Organisation for Scientific Research (NWO grant 916.76.077).

\section{Author details \\ ${ }^{1}$ Department of Neuroscience, NTNU, Trondheim, Norway. ${ }^{2}$ Department of Geriatrics, St. Olav University Hospital, Trondheim, Norway. ${ }^{3}$ Falls and Balance Research Group, Prince of Wales Medical Research Institute, University of New South Wales, Sydney, Australia. ${ }^{4}$ Department of Experimental-Clinical and Health Psychology, Faculty of Psychology and Educational Sciences, Ghent University, Belgium. ${ }^{5}$ Department of Rehabilitation Sciences and Physiotherapy, Faculty of Medicine and Health Sciences, Ghent University, Belgium. ${ }^{6}$ Research Institute MOVE, Faculty of Human Movement Sciences, VU University, Amsterdam, the Netherlands.}

\section{Authors' contributions}

All authors have read and approved the final manuscript. JLH, and MP have taken part in designing and planning of the study, data collection, data analysis and in writing the manuscript. DLS, JM, KD and SRL have taken part in designing and planning of the study, data analysis and in writing the manuscript.

\section{Competing interests}

The authors declare that they have no competing interests.

Received: 9 January 2010 Accepted: 17 August 2010

Published: 17 August 2010

\section{References}

1. Adlerton AK, Moritz U, Moe-Nilssen R: Forceplate and accelerometer measures for evaluating the effect of muscle fatigue on postural control during one-legged stance. Physiother Res Int 2003, 8:187-99.

2. Pinsault N, Vuillerme N: Differential postural effects of plantar-flexor muscle fatigue under normal, altered and improved vestibular and neck somatosensory conditions. Exp Brain Res 2008, 191:99-107.

3. Kavanagh J, Morrison S, Barrett RS: Lumbar and cervical erector spinae fatigue elicit compensatory postural responses to assist in maintaining head stability during walking. J App/ Physiol 2006, 101:1118-26.

4. Parijat $P$, Lockhart TE: Effects of lower extremity muscle fatigue on the outcomes of slip-induced falls. Ergonomics 2008, 51:1873-84.

5. Kent-Braun JA: Skeletal muscle fatigue in old age: whose advantage? Exerc Sport Sci Rev 2009, 37:3-9.

6. Chung LH, Callahan DM, Kent-Braun JA: Age-related resistance to skeletal muscle fatigue is preserved during ischemia. J Appl Physiol 2007, 103:1628-35.

7. Lanza IR, Russ DW, Kent-Braun JA: Age-related enhancement of fatigue resistance is evident in men during both isometric and dynamic tasks. J Appl Physiol 2004, 97:967-75.

8. Hakkinen K: Neuromuscular fatigue and recovery in women at different ages during heavy resistance loading. Electromyogr Clin Neurophysiol 1995, 35:403-13.

9. Kent-Braun JA, Ng AV, Doyle JW, Towse TF: Human skeletal muscle responses vary with age and gender during fatigue due to incremental isometric exercise. J Appl Physiol 2002, 93:1813-23.

10. Lanza IR, Larsen RG, Kent-Braun JA: Effects of old age on human skeletal muscle energetics during fatiguing contractions with and without blood flow. J Physiol 2007, 583:1093-105.

11. Mademli $L$, Arampatzis A: Mechanical and morphological properties of the triceps surae muscle-tendon unit in old and young adults and their interaction with a submaximal fatiguing contraction. J Electromyogr Kinesiol 2008, 18:89-98.

12. Rubinstein $\mathrm{S}$, Kamen $\mathrm{G}$ : Decreases in motor unit firing rate during sustained maximal-effort contractions in young and older adults. $J$ Electromyogr Kinesiol 2005, 15:536-43.

13. Schwendner Kl, Mikesky AE, Holt WS Jr, Peacock M, Burr DB: Differences in muscle endurance and recovery between fallers and nonfallers, and between young and older women. J Gerontol A Biol Sci Med 1997, 52: M155-60.

14. Yamada H, Okada M, Oda T, Nemoto S, Shiozaki T, Kizuka T, et al: Effects of aging on EMG variables during fatiguing isometric contractions. J Hum Ergol (Tokyo) 2000, 29:7-14

15. Allman BL, Cheng AJ, Rice CL: Quadriceps fatigue caused by catchlikeinducing trains is not altered in old age. Muscle Nerve 2004, 30:743-51.

16. Allman $B L$, Rice $C L$ : An age-related shift in the force-frequency relationship affects quadriceps fatigability in old adults. J Appl Physiol 2004, 96:1026-32.

17. Nussbaum MA: Effects of age, gender and task parameters on fatigue development during intermittent isokinetic torso extensions. Int J Industrial Ergonomics 2009, 39:185-91.

18. Davidson BS, Madigan ML, Nussbaum MA, Wojcik LA: Effects of localized muscle fatigue on recovery from a postural perturbation without stepping. Gait Posture 2009, 29:552-7.

19. Lin D, Nussbaum MA, Seol H, Singh NB, Madigan ML, Wojcik LA: Acute effects of localized muscle fatigue on postural control and patterns of recovery during upright stance: influence of fatigue location and age. Eur J Appl Physiol 2009, 106:425-34.

20. Mademli L, Arampatzis A, Karamanidis K: Dynamic stability control in forward falls: postural corrections after muscle fatigue in young and older adults. Eur J Appl Physiol 2008, 103:295-306. 
21. Petrella JK, Kim JS, Tuggle SC, Hall SR, Bamman MM: Age differences in knee extension power, contractile velocity, and fatigability. J Appl Physiol 2005, 98:211-20.

22. Helbostad JL, Leirfall S, Moe-Nilssen R, Sletvold O: Physical fatigue affects gait characteristics in older persons. J Gerontol A Biol Sci Med Sci 2007 62:1010-5.

23. Bellew JW, Fenter PC: Control of balance differs after knee or ankle fatigue in older women. Arch Phys Med Rehabil 2006, 87:1486-9.

24. Moore JB, Korff T, Kinzey SJ: Acute effects of a single bout of resistance exercise on postural control in elderly persons. Percept Mot Skills 2005, 100:725-33.

25. Nussbaum MA, Iridiastadi $H$, Wojcik LA: The influence of age on isometric endurance and fatigue is muscle dependent: a study of shoulder abduction and torso extension. Ergonomics 2007, 50:26-45.

26. Casale R, Rainoldi A, Nilsson J, Bellotti P: Can continuous physical training counteract aging effect on myoelectric fatigue? A surface electromyography study application. Arch Phys Med Rehabil 2003, 84:513-7.

27. MCNeil CJ, Rice CL: Fatigability is increased with age during velocitydependent contractions of the dorsiflexors. J Gerontol A Biol Sci Med Sci 2007, 62:624-9.

28. Stackhouse SK, Stevens JE, Lee SC, Pearce KM, Snyder-Mackler L, BinderMacleod SA: Maximum voluntary activation in nonfatigued and fatigued muscle of young and elderly individuals. Phys Ther 2001, 81:1102-9.

29. Alexander NB, Taffet GE, Horne FM, Eldadah BA, Ferrucci L, Nayfield S, Studenski S: Bedside-to-Bench Conference: Research Agenda for Idiopathic Fatigue and Aging. JAGS 2010, 58:967-75.

30. Pline KM, Madigan ML, Nussbaum MA, Grange RW: Lumbar extensor fatigue and circumferential ankle pressure impair ankle joint motion sense. Neurosci Lett 2005, 390:9-14.

31. Hicks AL, Mccartney N: Gender differences in isometric contractile properties and fatigability in elderly human muscle. Can J Appl Physiol 1996, 21:441-54.

\section{Pre-publication history}

The pre-publication history for this paper can be accessed here: http://www.biomedcentral.com/1471-2318/10/56/prepub

doi:10.1186/1471-2318-10-56

Cite this article as: Helbostad et al:: Consequences of lower extremity and trunk muscle fatigue on balance and functional tasks in older people: A systematic literature review. BMC Geriatrics 2010 10:56.

\section{Submit your next manuscript to BioMed Central and take full advantage of:}

- Convenient online submission

- Thorough peer review

- No space constraints or color figure charges

- Immediate publication on acceptance

- Inclusion in PubMed, CAS, Scopus and Google Scholar

- Research which is freely available for redistribution

Submit your manuscript at www.biomedcentral.com/submit
C Biomed Central 